\title{
Comment on "Spin crossover in (Mg,Fe)O: A Mössbauer effect study with an alternative interpretation of x-ray emission spectroscopy data"
}

\author{
György Vankó \\ European Synchrotron Radiation Facility, Boîte Postale 220, F-38043 Grenoble Cedex 9, France \\ Frank M. F. de Groot \\ Department of Inorganic Chemistry and Catalysis, Utrecht University, Sorbonnelaan 16, 3584 CA Utrecht, The Netherlands
}

(Received 15 May 2006; revised manuscript received 6 October 2006; published 7 May 2007)

\begin{abstract}
In a recent paper, Kantor et al. presented Mössbauer spectroscopy data on the pressure dependence of the $\mathrm{Fe}$ spin state in $\mathrm{Mg}_{1-x} \mathrm{Fe}_{x} \mathrm{O}$ [Phys. Rev. B 73, 100101(R) (2006)] and found that the transition was completed at significantly higher pressures than what had been found by x-ray emission spectroscopy (XES) studies. In order to account for the discrepancy, the authors reanalyzed the XES spectra using a simple spectral decomposition and achieved good agreement for the two types of data. In this paper, we show that the proposed alternative analysis of the XES spectra is inadequate, prove that previous XES work had correctly identified the low-spin state at the highest pressures, and provide a complete reanalysis of the XES data.
\end{abstract}

DOI: $10.1103 /$ PhysRevB.75.177101

PACS number(s): 75.30.Wx, 76.80.+y, 78.70.En

It has long been known that $\mathrm{x}$-ray emission spectroscopy (XES) is directly sensitive to the spin state of transition metals, ${ }^{1}$ and now it is coming into use to study spin transitions. ${ }^{2-6}$ The most prominent variations of the $K \beta$ line shape are seen on the low-energy side of the main $K \beta_{1,3}$ line: a satellite, known as $K \beta^{\prime}$, changes its intensity with spinstate transitions, which makes it easy to follow such transitions qualitatively. Extracting the total spin momentum of the $3 d$ electrons, however, is not straightforward due to the complexity of the numerous transitions that make up the spectra. The energies of the several multiplet terms are closely distributed in energy, there are no energy regions with complete spin polarization, and the arising lines are broad and strongly overlapping. Moreover, variation of the $3 d$ spin momentum leads to a completely new pattern of multiplet terms, so the spin-state variation affects the full spectral envelop. Therefore, fitting the spectra with any well-grounded theoretical model seems impossible. Several attempts were made to fit the spectra with a minimal set of established functions (Lorentzians, Voigt curves), but, possibly apart from the fortunate case of Mn where the satellite is very well separated, ${ }^{7}$ the resulting phenomenological descriptions of the line shape can be hardly applied to quantitative probing of spin transitions. 8,9 Consequently, other approaches, based on quantifying the variations of the satellite intensity ${ }^{2,6}$ or the full line shape $\mathrm{e}^{10-12}$ from difference spectra, are used, with increasing confidence. In a recent paper, Kantor et al. presented Mössbauer spectroscopy data on the pressure dependence of the $\mathrm{Fe}$ spin state in $\mathrm{Mg}_{1-x} \mathrm{Fe}_{x} \mathrm{O},{ }^{13}$ and found the transition pressure range significantly higher than what had been found with XES. 5,6 To account for the discrepancy, the authors reanalyzed the XES spectra of Ref. 6 using a simple spectral decomposition by fitting the spectra with the sum of an asymmetric Pearson IV and a Gaussian, and obtained agreement for the two types of data.

In this paper, we show that this proposed alternative analysis of the XES spectra is inadequate by applying it to another $\mathrm{Fe}(\mathrm{II})$ spin transition. We point out the causes of its failure, offer a more sound analysis, and reanalyze the XES data of Lin et al. ${ }^{6}$
We have studied $\left[\mathrm{Fe}(\text { phen })_{2}(\mathrm{NCS})_{2}\right] \quad($ phen $=1,10-$ phenantroline), a compound that exhibits an abrupt thermal spin transition at $185 \mathrm{~K}$, well above and below its transition temperature, with XES to secure the line shapes for pure high-spin (HS) and low-spin (LS) states. Crystal-field multiplet calculations were also performed to assist the XES lineshape analysis, while Mössbauer spectra were taken at the same temperatures $(295$ and $80 \mathrm{~K})$ to verify that the spin conversion is complete in both cases in the sample.

First, we show with the help of the XES spectra displayed in Fig. 1 that analysis in terms of two features, main line and satellite, is insufficient. Although the most conspicuous change is indeed seen on the satellite region, the underlying multiplet structure unveiled by theory reflects the very complex nature of the changes. As shown on the right of Fig. 1, many (around 250) multiplet terms of different intensities
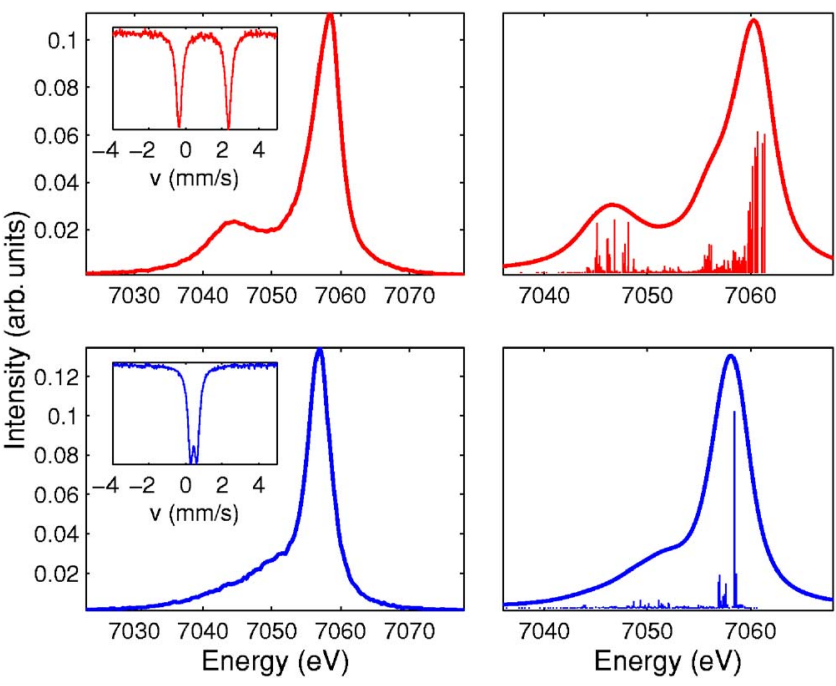

FIG. 1. (Color online) Experimental (left panel) and theoretical (right panel) $K \beta$ spectra of $\left[\mathrm{Fe}(\text { phen })_{2}(\mathrm{NCS})_{2}\right]$, in its HS (top) and LS (bottom) forms. Insets in the experimental part display the Mössbauer spectra. 
make up the spectra. The multiplet spectra have been calculated with the crystal-field multiplet model assuming a $1 s^{1} 3 d^{6}$ intermediate state for $\mathrm{Fe}(\mathrm{II})$. This model has been shown to be sufficient for a general simulation of the K XES spectral shapes of transition-metal compounds. ${ }^{14}$ More detailed simulations include charge-transfer effects and the inclusion of the $1 s$ XPS spectral shape for the excitation process. ${ }^{15,16}$ In addition, each transition can have a different linewidth due to the term-dependent lifetime broadening. ${ }^{17}$ The variation of the spin leads to a completely new pattern of multiplet terms, which results in the change of the full spectral envelop. Consequently, the LS and HS spectra have nothing in common, as it is very well illustrated by the multiplet structure; thus, the classification of the spectrum parts to satellite and main line should remain at a qualitative level. This is further supported by the width of the main line, which is narrower in the LS state.

The spectral changes at spin transition are not due to gradual changes at the iron sites. Instead, as Mössbauer spectroscopy clearly shows, in the transition regime both spin states are present: the spectra are superposition of those of the HS and LS, and the main change is seen in their intensity that follows their relative amount. ${ }^{18}$ This is not characteristic for molecular systems: coexistence of the two spin states has also been observed in oxides at pressure-induced spin transitions. ${ }^{19}$ While the Mössbauer parameters of each spinstate component can modestly vary at the transition due to the extreme sensitivity of the technique-not only to the distribution of the $3 d$ electrons but also to the lattice changesthe line patterns of XES, a very local probe, remain the same. We should also note that (at least in systems where there is a drop in the volume when going to the LS state from the HS) the crystal field does not change linearly at the transition, but jumps to that of the other state, typically from around 1.5 to $2.5 \mathrm{eV}$ in the case of $\mathrm{Fe}(\mathrm{II}){ }^{20}$ This can explain why the spectra should not change in a continuous way. Moreover, it has been shown by theory that even large crystal-field variations barely affect the XES line shape as long as the spin state remains the same. ${ }^{21}$ Accordingly, in a high-pressure XES experiment on $\mathrm{CoO}$ identical line shapes were obtained for pressures between 0 and $140 \mathrm{GPa}^{22}$ Therefore, the spectra should be decomposed using the full HS and LS line shapes. This can be accomplished by fitting, if proper (good statistical quality, without artificial shifts or extra broadenings) reference spectra are available, or by comparing the integrals of the absolute values of the difference spectra (IAD) to that obtained on reference data. ${ }^{11,12}$

The spectra of Fig. 2, which comprises two intermediate spectra $(\mathrm{spA}, \mathrm{spB})$ besides the two proper reference ones (HS, LS) without any artificial energy shifts, provide ideal grounds for testing line-shape analysis methods. To start with, the intermediate spectra were fitted with the sum of the reference ones, the only fit parameter being the HS fraction. The fits give $100 \%, 72.5 \%, 41.8 \%$, and $0 \%$ HS fractions for the spectra HS, spB, spA, and LS.

As seen on the spectra, besides the intensity changes of the satellite, a relevant apparent energy shift is also present. These two effects result in large overall spectral differences, shown below the spectra, where difference curves from that of the LS are also shown. These spectral differences consist

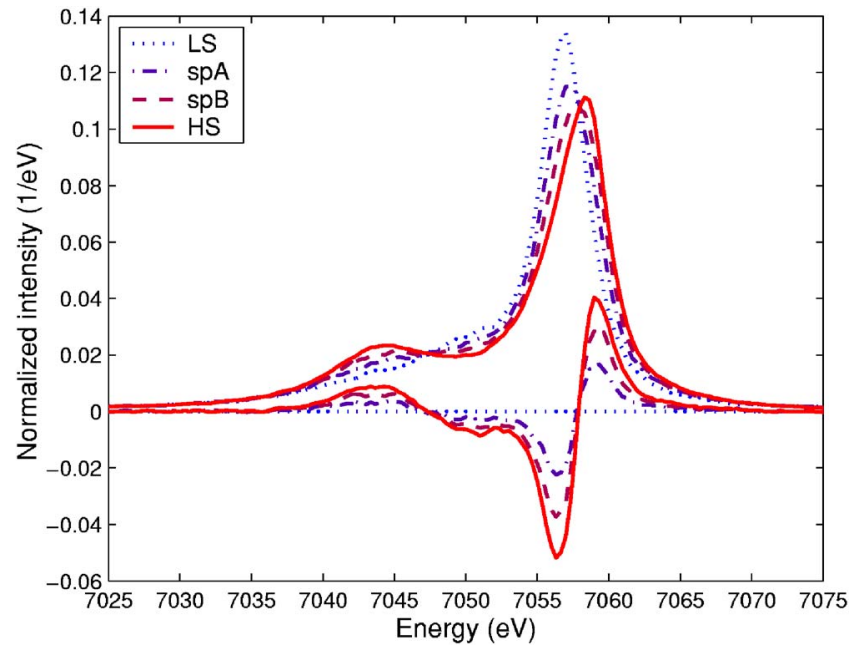

FIG. 2. (Color online) Four spectra on the spin transition of $\left[\mathrm{Fe}(\mathrm{phen})_{2}(\mathrm{NCS})_{2}\right]$. Differences from the LS line shape are also shown below the spectra.

the main ingredients of the above-mentioned IAD method we have suggested recently to evaluate the total $3 d$ spin momentum from XES. ${ }^{11,12}$ The IAD approach gives $100 \%$, $72.1 \%, 42.2 \%$, and $0 \%$ HS fractions, in excellent agreement with the fits. Another important observation is that identical center of mass was found for all spectra. This justifies the proposition of Glatzel and Bergmann who suggest shifting the spectra to have the same center-of-mass energy when the energy scale is uncertain. ${ }^{23}$

Kantor et al. proposed fitting the HS spectrum with a Pearson IV and a Gaussian, then fixing the width and asymmetry of the Pearson and fitting the other spectra with this and the Gaussian; the intensity of the latter was proposed to be proportional to the average spin. ${ }^{13}$ Applying this analysis, we obtained Gaussian areas for "satellite peak intensities" of $15.2 \%, 13.3 \%, 10.5 \%$, and $7.1 \%$ (in terms of spectral area) for the spectra HS, $\mathrm{spB}, \mathrm{spA}$, and LS. This corresponds to HS fractions of $100 \%, 87.6 \%, 69.5 \%$, and $47 \%$ (or $S=2$, $1.75,1.39$, and 0.94). This implies that for the LS spectrum this approach erroneously shows that half of the iron in the sample is in the HS state, and the intermediate spectra are also interpreted to have bigger HS contribution than they should. When looking into the causes of the big discrepancy with the analysis of Kantor et al., several reasons can be identified on why the latter fails.

First of all, the sum of a Pearson IV and a Gaussian seems far too simple for the multiplet structure. Although the fits might be reproducing the line shape well, no justification is given in Ref. 13 as to why this ad hoc spectral decomposition should also grasp the relevant physical aspects. Moreover, the main line cannot be described with a Pearson IV of identical linewidth and asymmetry: the LS and HS linewidths are different, and the energy shift causes apparent additional broadening in the transition region. Finally, the crucial assumption of Kantor et al. is that the Gaussian intensity vanishes when the pure LS spin state is reached; this implies that a single Pearson IV should be able to describe the LS line shape. This is not the case: the extra intensity on the low-energy side requires a relevant Gaussian intensity, as 


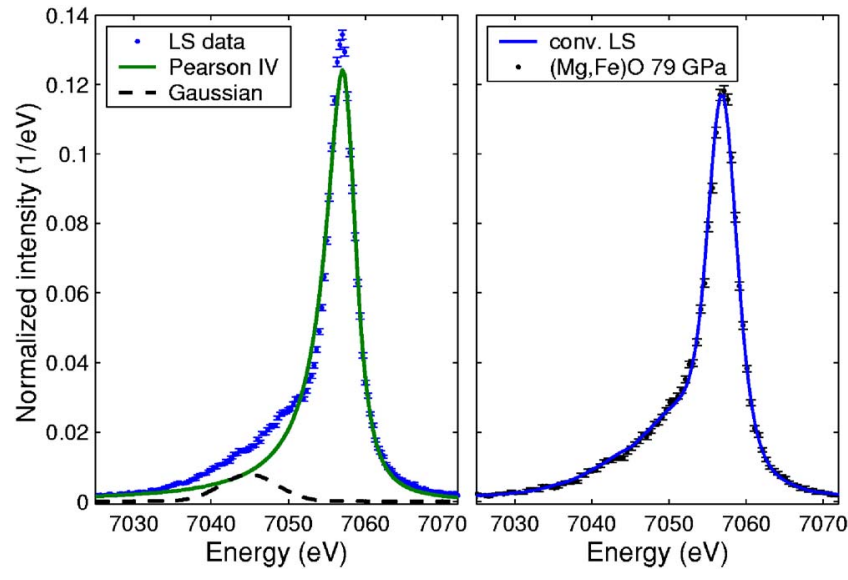

FIG. 3. (Color online) Left: fitting of the LS data with the model of Kantor et al. Right: the LS data convoluted with a Gaussian of $2.2 \mathrm{eV}$ FWHM compares well with the $79 \mathrm{GPa} \mathrm{Mg}_{0.75} \mathrm{Fe}_{0.25} \mathrm{O}$ spectrum from Ref. 6.

seen in the left of Fig. 3. Releasing all fit constraints (and leaving out the Gaussian) results in an almost identical Pearson line (which is left out from the figure as at this size would not be fully distinguishable). The main mistake in the analysis of Kantor et al. is that it was not checked whether the approach could describe LS line shapes.

We also wish to point out that using the energy difference of the main peak and the satellite, denoted as $\Delta E$ in Ref. 13, is an artificial quantity when obtained on apparent spectral features in the transition region, where the shape of the spectrum depends on the superposition coefficients only. Consequently, this cannot give further physical insights as the authors claim. Moreover, the variations of the total spectral area, used in Ref. 13 to find correlation with the satellite intensity variations, reveal only incorrect normalization of the spectra: the area should remain the same, as the overall $K \beta$ emission yield does not depend on the spin state.

As shown above, the analysis of Kantor et al. results in $S \approx 1$ for the LS sample. From the foregoing, it is not surprising that this coincides with the value obtained with the same analysis on $(\mathrm{Mg}, \mathrm{Fe}) \mathrm{O}$ at the highest pressure. ${ }^{13}$ This suggests that the spectral shapes are similar. Indeed, as we can see in the right of Fig. 3, convolution of the spectrum of the LS reference with a Gaussian of $2.2 \mathrm{eV}$ full width at half maximum (FWHM) perfectly matches the $79 \mathrm{GPa}$ $\mathrm{Mg}_{0.75} \mathrm{Fe}_{0.25} \mathrm{O}$ spectrum from Ref. 6. This line-shape similarity is a strong argument that the spin state is the same $(S=0)$ in the two cases; the different broadening can stem from the differences in the experimental conditions. Moreover, at the highest three pressure points, no further variations are observed on the spectra, which suggests that the spin transition to the LS state is complete.

Finally, we reanalyze the XES data of Lin et al. published in Ref. 6. As the raw spectra had different background levels, a constant background is subtracted. More importantly, the spectra are normalized to the area and shifted to the center of mass, thus, each spectrum has the same zeroth and first moments. We saw that the spectrum taken at the highest pressure reflects iron in the LS state; the one obtained at ambient

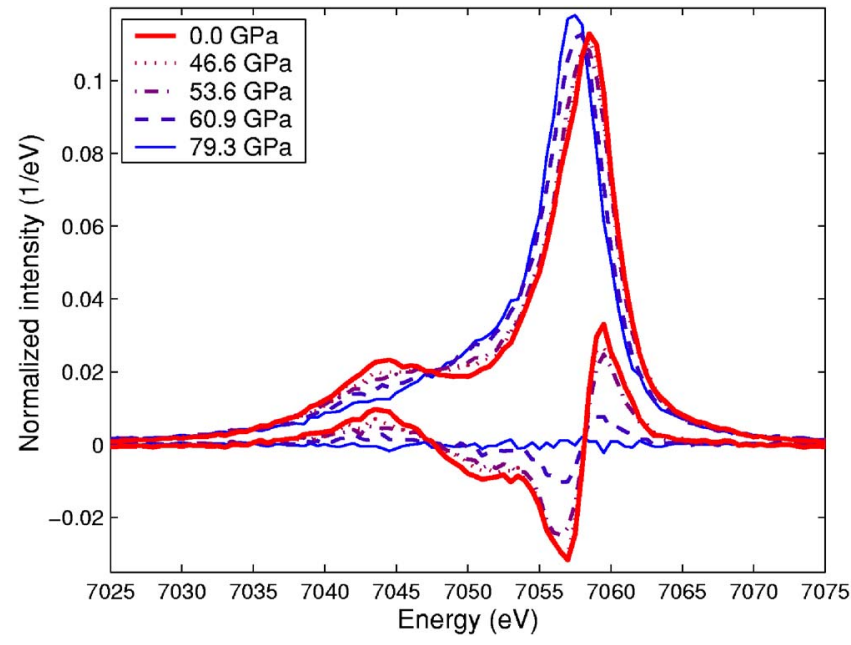

FIG. 4. (Color online) Selected spectra reflecting the spin transition in $\mathrm{Mg}_{0.75} \mathrm{Fe}_{0.25} \mathrm{O}$ from Ref. 6. Differences from the LS reference are also shown.

conditions has HS iron; thus, we have proper (inner) references, which allows the IAD analysis. To create the difference spectra, we subtracted from each the average of those taken at the highest three pressures, which were the same within the error. This was advantageous as the individual spectra have limited statistical quality. The IAD values obtained by integration of the absolute values of the difference spectra were projected to the $S=0, \ldots, 2$ scale. Some of the spectra and the differences are shown in Fig. 4; the results extracted on the average spin are plotted in Fig. 5, together with the analysis of Kantor et al. and Lin et al. It is apparent from the latter figure that our analysis, although providing smaller standard deviations and thus presumably more accurate values due to the larger area differences used in the evaluation, eventually secures the same transition pressure as that of Lin et al. (Similar conclusions can be drawn for the analysis of the high-pressure XES data of $\mathrm{Mg}_{0.40} \mathrm{Fe}_{0.60} \mathrm{O}$, see Ref. 24 for details.) The IAD values for the HS spectra are 0.291 for $\mathrm{Mg}_{0.75} \mathrm{Fe}_{0.25} \mathrm{O}$ and 0.321 for $\left[\mathrm{Fe}(\text { phen })_{2}(\mathrm{NCS})_{2}\right]$. This agreement is notable; the $10 \%$ difference is not too

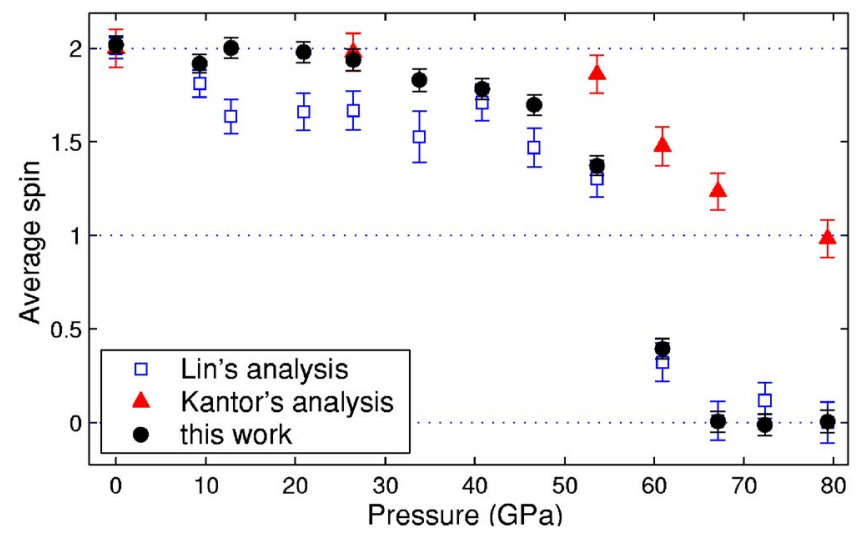

FIG. 5. (Color online) Comparison of the different analyses of the $\mathrm{Mg}_{0.75} \mathrm{Fe}_{0.25} \mathrm{O}$ XES data. 
large considering that these numbers were obtained on spectra collected on different spectrometers, at different experimental conditions (and broadenings), and on samples of quite different natures. This raises the prospect that the IADspin correlations are transferable for systems with the same transition-metal ions. Moreover, in the possession of this number, the IAD can also give the spin variation between two experimental points without the need of reference spectra.

In conclusion, we showed that the XES analysis proposed by Kantor et al. is unfounded. We have also tested a very recently developed analysis method on a reference spintransition system and applied to the same high-pressure data, too. This and other recent works show that XES with careful data analysis is becoming established as a quantitative technique that can procure the $3 d$ spin momentum. While in the case of iron XES needs more effort in general to compete with the accuracy of Mössbauer spectroscopy, it can be extended to other transition metals still keeping the benefit of element selectivity, where the isotope specific Mössbauer spectroscopy cannot be applied; a further advantage is that the technique is not influenced by magnetic order or magnetic fluctuations and relaxations. Last of all, we agree with the authors of Ref. 13 that Mössbauer spectroscopy and XES should reflect the same transition pressure. However, in the case of $\mathrm{Mg}_{0.75} \mathrm{Fe}_{0.25} \mathrm{O}$, according to a very recent synchrotron Mössbauer spectroscopy study, ${ }^{25}$ the transition to the LS state is completed between 52 and $70 \mathrm{GPa}$, in agreement with the XES data. ${ }^{5,6}$

The authors thank J.-F. Lin for kindly sharing his data. J. Badro, J.-F. Lin, G. Monaco, and J.-P. Rueff are gratefully acknowledged for their careful and critical reading of the paper.
${ }^{1}$ K. Tsutsumi, H. Nakamori, and K. Ichikawa, Phys. Rev. B 13, 929 (1976).

${ }^{2}$ J.-P. Rueff, C.-C. Kao, V. V. Struzhkin, J. Badro, J. Shu, R. J. Hemley, and H. K. Mao, Phys. Rev. Lett. 82, 3284 (1999).

${ }^{3}$ J. Badro, V. V. Struzhkin, J. Shu, R. J. Hemley, H.-K. Mao, C.-C. Kao, J.-P. Rueff, and G. Shen, Phys. Rev. Lett. 83, 4101 (1999).

${ }^{4}$ G. Vankó, T. Neisius, F. Renz, S. Kárpáti, A. Shukla, A. Mirone, and F. de Groot, Highlights ESRF 2002 (http://www.esrf.fr/ UsersAndScience/Publications/Highlights/2002/HRRS/ HRRS9/), p. 59.

${ }^{5}$ J. Badro, G. Fiquet, F. Guyot, J.-P. Rueff, V. V. Struzhkin, G. Vankó, and G. Monaco, Science 300, 789 (2003).

${ }^{6}$ J.-F. Lin, V. V. Struzhkin, S. D. Jacobsen, M. Y. Hu, P. Chow, J. Kung, H. Liu, H.-K. Mao, and R. J. Hemley, Nature (London) 436, 377 (2005).

${ }^{7}$ T. A. Tyson, Q. Qian, C.-C. Kao, J.-P. Rueff, F. M. F. de Groot, M. Croft, S. W. Cheong, M. Greenblatt, and M. A. Subramanian, Phys. Rev. B 60, 4665 (1999).

${ }^{8}$ G. Hölzer, M. Fritsch, M. Deutsch, J. Härtwig, and E. Förster, Phys. Rev. A 56, 4554 (1997).

${ }^{9}$ S. D. Gamblin and D. S. Urch, J. Electron Spectrosc. Relat. Phenom. 113, 179 (2001).

${ }^{10}$ J. P. Rueff, A. Shukla, A. Kaprolat, M. Krisch, M. Lorenzen, F. Sette, and R. Verbeni, Phys. Rev. B 63, 132409 (2001).

${ }^{11}$ G. Vankó, J.-P. Rueff, A. Mattila, Z. Németh, and A. Shukla, Phys. Rev. B 73, 024424 (2006).

${ }^{12}$ G. Vankó, T. Neisius, G. Molnár, F. Renz, S. Kárpáti, A. Shukla, and F. M. F. de Groot, J. Phys. Chem. B 110, 11647 (2006).

${ }^{13}$ I. Y. Kantor, L. S. Dubrovinsky, and C. A. McCammon, Phys.
Rev. B 73, 100101(R) (2006).

${ }^{14}$ G. Peng, F. de Groot, K. Hämäläinen, J. Moore, X. Wang, M. Grush, J. Hastings, D. Siddons, W. Amstrong, O. Mullins et al., J. Am. Chem. Soc. 116, 2914 (1994).

${ }^{15}$ F. M. F. de Groot, Chem. Rev. (Washington, D.C.) 101, 1779 (2001).

${ }^{16}$ P. Glatzel, U. Bergmann, F. M. F. de Groot, and S. P. Cramer, Phys. Rev. B 64, 045109 (2001).

${ }^{17}$ M. Taguchi, T. Uozumi, and A. Kotani, J. Phys. Soc. Jpn. 66, 247 (1997).

${ }^{18}$ P. Ganguli, P. Gütlich, and E. W. Müller, Inorg. Chem. 21, 3429 (1982).

${ }^{19}$ M. P. Pasternak, G. K. Rozenberg, W. M. Xu, and R. D. Taylor, High Press. Res. 24, 33 (2004).

${ }^{20}$ P. Gütlich, A. Hauser, and H. Spiering, Angew. Chem., Int. Ed. Engl. 33, 2024 (1994).

${ }^{21}$ X. Wang, F. M. F. de Groot, and S. P. Cramer, Phys. Rev. B 56, 4553 (1997).

${ }^{22}$ J.-P. Rueff, A. Mattila, J. Badro, G. Vankó, and A. Shukla, J. Phys.: Condens. Matter 17, S717 (2005).

${ }^{23}$ P. Glatzel and U. Bergmann, Coord. Chem. Rev. 249, 65 (2005).

${ }^{24}$ See EPAPS Document No. E-PRBMDO-75-084713 for details on the analysis of the $\mathrm{Mg}_{0.40} \mathrm{Fe}_{0.60} \mathrm{O}$ XES. This document can be reached via a direct link in the online article's HTML reference section or via the EPAPS homepage (http://www.aip.org/ pubservs/epaps.html).

${ }^{25}$ J.-F. Lin, A. G. Gavriliuk, V. V. Struzhkin, S. D. Jacobsen, W. Sturhahn, M. Y. Hu, P. Chow, and C.-S. Yoo, Phys. Rev. B 73, 113107 (2006). 